\title{
Visual exploration pattern in hemineglect
}

\author{
René M. Müri · D. Cazzoli · T. Nyffeler · T. Pflugshaupt
}

Received: 27 January 2008 / Accepted: 18 September 2008 / Published online: 16 December 2008

(C) Springer-Verlag 2008

\begin{abstract}
The analysis of eye movement parameters in visual neglect such as cumulative fixation duration, saccade amplitude, or the numbers of saccades has been used to probe attention deficits in neglect patients, since the pattern of exploratory eye movements has been taken as a strong index of attention distribution. The current overview of the literature of visual neglect has its emphasis on studies dealing with eye movement and exploration analysis. We present our own results in 15 neglect patients. The free exploration behavior was analyzed in these patients presenting 32 naturalistic color photographs of everyday scenes. Cumulative fixation duration, spatial distribution of fixations in the horizontal and vertical plane, the number and amplitude of exploratory saccades was analyzed and compared with the results of an age-matched control group. A main result of our study was that in neglect patients, fixation distribution of free exploration of natural scenes is not only influenced by the left-right bias in the horizontal direction but also by the vertical direction.
\end{abstract}

\section{Introduction}

Neglect can generally be defined as failure to attend to the contralesional side of space (Buxbaum et al., 2004). Patients suffering from neglect behave as if the contralesional side of the world would not exist anymore (Karnath,

R. M. Müri $(\bowtie) \cdot$ D. Cazzoli · T. Nyffeler · T. Pflugshaupt Perception and Eye Movement Laboratory,

Department of Neurology,

Department of Clinical Research,

Inselspital, Bern University Hospital,

University of Bern, 3010 Bern, Switzerland

e-mail: rene.mueri@dkf.unibe.ch
Himmelbach, \& Rorden, 2002). Neglect is frequent, involving approximately $13-81 \%$ of patients with right-hemispheric stroke (Barrett et al., 2006). The symptoms are supramodal and involve vision, motor activity, tactile sensation, hearing, or olfaction. They cannot be explained by elementary dysfunction of a modality (Parton, Malhotra, \& Husain, 2004). Moreover, neglect may affect different spatial planes (personal, peripersonal, extrapersonal, representational) and may be space- or object-centered (i.e., referring to an egocentric or object-centered reference frame), appearing in dissociated forms or in combination (Buxbaum et al., 2004).

Neglect is often associated with other signs. Among the most frequent, general reduction of attentional arousal, hemianopia, anosognosia, and extinction phenomena have been described (Parton et al., 2004). Neglect is more often found after right than left hemispheric lesions, commonly associated with extensive strokes of the middle cerebral artery (Parton et al., 2004). However, lesions of different brain regions may result in neglect. The angular and supramarginal gyrus of the right-inferior parietal lobe has been described as critical for neglect. Furthermore, more lateral structures such as the parieto-temporal junction or the superior temporal gyrus may play an important role (Vallar, 2001; Parton et al., 2004), and lesions of the frontal lobe including the inferior and middle frontal gyrus have been reported to result in neglect (Husain \& Kennard, 1996; Vallar, 2001; Parton et al., 2004). Frontal lobe lesions may provoke more transient neglect syndromes (Parton et al., 2004).

Two recent studies have specifically addressed the problem concerning critical cortical regions. In their analysis, Mort etal. (2003) investigated patients suffering from neglect after stroke in the areas supplied by the medial or the posterior cerebral artery. For patients with medial cerebral 
artery stroke, the neglect-critical area was identified in the angular gyrus of the inferior parietal lobe, thus being consistent with regions commonly assumed as crucial. For patients with posterior cerebral artery stroke, the relevant lesion area was located more medially and deep in the parahippocampal region of the temporal lobe. The second study (Karnath, Fruhmann-Berger, Küker, \& Rorden, 2004) yielded different results. Their analysis revealed that the structure most common damaged in neglect patients was the superior temporal gyrus. In contradiction to the commonly assumed primacy of parietal regions, this study replicated the findings of a previous study by the same research group (Karnath, Ferber, \& Himmelbach, 2001).

The superior temporal gyrus was also damaged in a large part of the patients included in the study by Mort et al. (2003). As stated by Parton et al. (2004), it is therefore possible that this region is important but not fundamental for neglect onset. Furthermore, not only cortical lesions provoke neglect, but also lesions to the underlying white matter and subcortical structures. Lesions in the white matter may damage connections, thus disrupting corresponding neural networks (Vallar, 2001). The putamen, pulvinar and caudate nucleus have been identified as crucial subcortical structures leading to neglect (Karnath et al., 2002). Other studies reported neglect after thalamic lesions (e.g., Karussis, Leker, \& Abramsky, 2000).

Recent accounts seem to converge towards a concept of neglect as a multi-component syndrome with neglect subtypes (e.g., Marshall, Halligan, \& Robertsin, 1993; Chatterjee, 1998; Danckert \& Ferber, 2006).

\section{Approaches to visuospatial neglect}

In the literature, different approaches have been suggested to explain neglect. Each approach is based on certain components of the neglect symptomatology and postulates one or more alternative models.

\section{Attentional models}

The neglect model proposed by Kinsbourne (1993) is based on the assumption that two opponent processors, located in the left and right hemispheres, control attention. These processors direct attention towards the most eccentric contralateral portion of space. They reciprocally inhibit their effects, thus the actual direction of attention is represented by a gradient, the vectorial result of their interactions. According to this model, unilateral neglect is a consequence of a hemispherical imbalance. When one hemisphere is lesioned, the intact one biases attention towards the ipsilesional side, since the inhibition from the damaged hemisphere is no longer effective. Kinsbourne's model predicts two different abnormal attentional gradients for the two visual hemifields. The contralesional hemifield would show a gradient of decreasing attentional performance from the center to the periphery. An inverted gradient would increase from center to periphery in the ipsilesional hemifield. The assumptions of the orientational bias model are primarily supported by the observation of patients' performance in clinical tests such as line bisection. Further evidence comes from reaction time measurements. Smania et al. (1998) studied the distribution of visual attention asking neglect patients and normal control subjects to manually respond to small flashing lights positioned at different peripheral locations on a horizontal arc perimeter. Participants performed the task maintaining central gaze fixation, thus using peripheral vision. The results indicated higher detection error rates and reaction times in the contralesional than in the ipsilesional hemifield. Moreover, neglect patients exhibited a gradient of increasing error rates from center to periphery in the contralesional hemifield and a moderate difference between the most central and the other more peripheral positions in the ipsilesional hemifield, the latter being slightly better and detected more quickly. These results suggest a hyperattention towards the ipsilesional hemifield, supporting the hemispherical imbalance theory proposed by Kinsbourne.

The question about the integrity (or the enhancement) of the attentional performance in the right hemisphere has also been specifically addressed by more recent studies. Bartolomeo and Chokron (1999a) analyzed the reaction times of manual responses to left and right targets in patients with neglect symptoms of different severity. They found a correlation between severity of symptoms and reaction times. The regression curve for left-sided targets was steeper than the one for right-sided targets, indicating an attentional bias towards the right. Nevertheless, this bias is characterized by impaired and not by enhanced attention. The model of unilateral neglect proposed by Posner, Walker, and Friedrich. (1984) is based on the concept of attentional shift as a sequence of three basic internal mechanisms: (1) disengagement of attention from the current focus, (2) moving attention towards the new target, and (3) engagement of attention onto the target. According to this model, parietal lesions would cause a selective impairment of the disengagement mechanism causing difficulties in redirecting attention towards the contralesional side. The model also posits a disruption of attentional orientation, with the consequence of a deficit in shifting towards the contralesional side (i.e., increased latencies). The assumptions of this model are supported by reaction time tasks including cues presented with different stimulus onset asynchrony (SOA). Posner showed that a disengagement deficit for contralesional targets occurs after parietal lesions, but not after frontal, midbrain, or temporal lesions. Losier and Klein 
(2001) reviewed studies applying analogue paradigms in patients with left and right-parietal lesions, with or without neglect symptoms. Based on these studies they suggested that the disengagement deficit is consistent for peripheral but not for central cues. Furthermore, the disengagement deficit was inversely proportional to the SOA. The effects were enhanced in patients with neglect symptoms, but they were also present to a lesser extent in patients with left hemispherical lesions, raising some doubts about the specificity of the disengagement deficit for neglect.

\section{Representational theory of neglect}

Bisiach, Pizzamiglio, Nico, and Antonucci (1996) proposed a distorted and anisometric space representation along the horizontal dimension as the underpinnings of neglect. According to this model, the representational space shows a pathological expansion towards the contralesional side and a compression towards the ipsilesional one, following a logarithmic scale. An alternative model by Halligan and Marshall (1991) suggests that points of the left representational space are linearly and uniformly compressed rightwards along the horizontal axis. Bartolomeo et al. (2003) replicated the study by Halligan and Marshall and found similar results.

\section{Transformational theory of neglect}

The transformational theory (Karnath, 1997) suggests that the phenomenology of neglect is caused by an impairment of the transformation of sensory input into an egocentric frame of reference. Due to a systematic error, the egocentric space representation deviates towards the ipsilesional side, following a rotation around the vertical body axis. The assumptions of this model are primarily supported by several studies assessing neglect patients' behavioral patterns in visual exploration tasks. For example, in one study patients with neglect were requested (Karnath, Niemeier, \& Dichgans, 1998) to search for a (non-existent) target letter among a random array of other letters, presented on the inner surface of a sphere. Seated in the sphere, patients were free to perform eye and head search movements. Similar patterns for healthy participants and neglect patients were observed. Head and eye-in-head movements formed a symmetric, pseudo bell-shaped distribution around particular locations along the horizontal axis. In healthy controls, this location corresponded to the mid-sagittal plane of the body for head movements, and to the head midline for eyein-head movements. In neglect patients, the distribution was shifted to the right. Chokron and Bartolomeo (1997) asked neglect patients and right hemisphere damaged patients without neglect to point with their index finger to the position they felt as being 'straight ahead' while blindfolded. The results showed no significant correlation between errors in the task and presence of neglect and both patient groups displayed inconsistent deviations towards the right, the left, or no bias at all. A further study by the same authors (Bartolomeo \& Chokron, 1999b) demonstrated that the shifts in the straight ahead pointing tasks did not correlate with data from different clinical neglect tests or with accuracy and response times to lateralized stimuli. Deviation of the egocentric reference system in neglect was further investigated by Richard, Rousseaux, and Honoré (2005). In their experiment, patients were asked to perform the straight ahead pointing task in light or darkness, with free eye movements or stable gaze fixation on a central or peripheral visual target. The typical deviation in neglect patients was observed, and a good accuracy in the control group. However, the deviation of the subjective midline in neglect patients was more severe in light than in darkness, and the central fixation reduced the pointing error in this group only, suggesting that the observed deviation in the egocentric frame of reference is influenced by attentional factors.

Both representational and transformational approaches stress spatial features to explain visuospatial neglect. The former postulate a deformation of space representation, the latter a shift of its reference frame. However, a closer look at the supporting evidence for both approaches highlights substantially different operational approaches. In the representational approach, the concept of space is mainly tested in two dimensions and its spatial extent is limited (i.e., computer screen, sheet of paper, etc.). In the transformational approach, the tasks include the third dimension, the ambient space of the subject. Dissociations in the neglect phenomenology depending on the spatial plane are known (e.g., Parton et al., 2004). Summing up, it seems that none of the presented models alone can account for 'visuospatial neglect' as a unitary and superordinate concept. Furthermore, there is increasing evidence that also non-spatially lateralized mechanisms play an important role in hemispatial neglect (Husain \& Rorder, 2003).

\section{Eye movements, visual exploration, and attention}

When facing a visual scene, we are surrounded by a wide variety of stimuli reaching our visual system. Only a small part of the enormous information load reaches consciousness and influences interaction with the environment. On the one hand, it is important to select relevant information to direct our actions; on the other hand we have to keep track of the visual scene as a whole, in order to gain an overview of our surroundings. This complex regulation includes both top-down and bottom-up attentional processes. Top-down processes consist of concept-driven 
encoding and processing of stimuli, i.e., based on context, beliefs, desires, knowledge, etc. Conversely, bottom-up processes encompass data- or stimulus-driven mechanisms that are based on structural features of the stimuli such as color, brightness, orientation, etc. The complex interaction between these two processes has been described by Treisman and Gelade (1980) in their 'feature-integration theory of attention'.

Visual exploration is an essential aspect of perception. Human beings typically orient their attention across the surrounding space by means of eye movements (Himmelbach, Erb, \& Karnath, 2006). Detailed information analysis is only possible owing to the high resolution of the fovea, which must therefore be displaced towards the visual field regions where a more accurate processing is needed. This mechanism is called foveation and is equivalent to fixation, which is one of the two main phases of eye movements. Saccades are rapid ballistic eye movements that are necessary to displace the fovea towards the next foveation. During the execution of saccadic eye movements, practically no visual information can be gained. Taking together the above-mentioned concepts about visuospatial attention and eye movements, one could assume that measuring eye movements provides reliable information on the subjects' overt allocation of attention in space. Furthermore, functional imaging studies have shown that attention and eye movements share largely overlapping or common neural networks (Corbetta et al., 1998; Ignashchenkova, Dicke, Haarmeier, \& Thier, 2004; Nobre, Gitelman, Dias, \& Mesulam, 2000). Finally, central components of disorders typically characterized by deficient attention such as neglect could be reliably described and investigated by means of eye movements (e.g., Malhotra, Coulthard, \& Husain, 2006; Mort \& Kennard, 2003; Pflugshaupt et al., 2004; Sprenger, Kömpf, \& Heide, 2002).

\section{Visual exploration in neglect}

The visual exploration patterns in neglect patients closely reflect their relative inability to attend the contralesional side of space. In fact-although their visual file is often spared-such patients show a marked lack of active exploration of the contralesional side, their visual fixations and focus of attention being orientated towards the ipsilesional side (Sprenger et al., 2002). For instance, when asked to explore a picture on a screen, neglect patients' early attentional orienting is biased towards the ipsilesional side, and eye movements produce an asymmetrical pattern where the contralesional half of the picture is virtually spared. Moreover, visual exploration within the ipsilesional picture half is characterized by frequent re-fixations, i.e., recurrent returns to already explored areas (Mort \& Kennard, 2003).
Niemeier and Karnath (2000) found that saccades in neglect patients during free visual exploration are actually hypometric, but there are no specific differences between left-to-right and right-to-left saccades. Moreover, Walker and Findlay (1996) could demonstrate that-if directionspecific deficits in saccadic latency and length are present-they are rather a result than a causal factor of neglect. In fact, such saccadic impairments could be the consequence of what Malhotra et al. (2006) called "restricted effective field of vision". It is not surprising to find impairments in fixations and saccades in neglect patients, since the neural basis of spatial exploration includes regions which may play a central role in the neglect syndrome, such as the temporo-parietal junction, the superior temporal gyrus, the inferior frontal gyrus and-to a smaller extentthe inferior temporal lobe (Himmelbach et al., 2006).

For the two above-mentioned tendencies characterizing visual exploration in neglect patients, different explanations have been put forward, namely an asymmetry of fixation distribution favoring the contralesional hemifield, and an exploration pattern characterized by recurrent fixations within the latter. The asymmetrical fixation distribution can be interpreted in the framework of the different explanatory approaches for visuospatial neglect, which have been outlined before. For free exploration tasks, the predictions of Kinsbourne's (1993) attentional bias model and of Karnath's (1998) transformational model are particularly interesting. Many studies involving neglect patients and visual exploration with free head and eye movements have been realized by Karnath and collaborators (Karnath \& Fetter, 1995; Karnath, Fetter, \& Dichgans, 1996; Karnath et al., 1998). A stable pattern of results was found in these studies, namely that in neglect patients fixation distribution was symmetrical and bell-shaped as in healthy subjects, but shifted towards the ipsilesional side by approximately $15^{\circ}$ around an earth-vertical axis. These results may be in contradiction to models postulating a deformed space representation (Bisiach et al., 1996; Halligan \& Marshall, 1991) or to the orientational bias model by Kinsbourne (1993), postulating an increasing contralesional-to-ipsilesional gradient. Behrmann, Watt, Black, and Barton (1997) performed a comprehensive analysis of the eye movement patterns of neglect patients in a visual search task, finding a left-toright gradient. They found a gradient of fixation distribution with a displacement of the peak of the fixation distribution curve that was located at a medium eccentricity, is more consistent with the transformational account.

The observed increased rate of re-fixations within the right hemifield in neglect patients has been interpreted as hyperattention in the ipsilesional hemifield, deficient attentional disengagement from the latter, impaired transsaccadic spatial working memory (which does not permit to retain locations between saccades), compulsive motor 
perseveration, or deficient inhibition of return (Sprenger et al., 2002).

Heide and Kömpf (1998) showed that visual exploration impairment in neglect is dissociable depending on the lesion site. Patients with frontal lesions (in the area of the frontal eye field) showed a predominant impairment in a task involving intentional, serial exploration (i.e., of abstract, structured configurations), but not when exploring natural scenes. Patients with posterior parietal lesions displayed the inverse pattern. The authors concluded that frontal regions play a central role in voluntary exploration behavior, while parieto-temporal regions substantially contribute to reflexive orienting of attention.

\section{Neglect in the vertical plane (altitudinal neglect)}

In contrast to the horizontal bias in neglect, the consequences of neglect in the vertical plane have been rarely studied. Altitudinal neglect seems to occur after bilateral lesions. Rapcsak, Cimino, and Heilman (1988) reported a patient with bilateral parieto-occipital infarctions presenting altitudinal neglect. In a task to indicate the midpoint of a vertical oriented bar, the patient estimated the midpoint always above the true one (shift to the upper part of the extrapersonal space). Shelton, Bowers, and Heilman (1990) examined a patient with bilateral inferior temporal lobe infarction and found neglect for the far peripersonal space and the upper vertical space. Neglect for near peripersonal and for inferior space was described by Mennemeier, Wertman, and Heilman (1992) and by Mark and Heilman (1997). Thus, biparietal injury seems to be associated with neglect for the near and inferior space, while bilateral temporooccipital injury is associated with neglect of far and superior space (Adair, Williamson, Jacobs, \& Heilman, 1995).

Halligan and Marshall (1989) found in 26 unilateral neglect patients, that these patients made most errors in the lower quadrant of the array of a cancellation task, contralateral to the lesion. Recently, Cappelletti, Freeman, and Cipolotti (2007) used a mental number bisection task. In this task, neglect patients were asked to report the middle number between two numbers. They manipulated the number line orientation by changing the task instruction: neglect patients were told that the number-pairs represented either houses on a street (i.e., the horizontal condition) or floors in a building (vertical condition). All five tested neglect patients showed a rightward horizontal bias, but only three of the patients also showed an upward bias for vertically oriented number lines, suggesting that horizontal and vertical neglect can associate or dissociate among different patients.

To study free visual exploration behavior in the horizontal and vertical plane in neglect patients, we tested 15 patients with acute left hemineglect. The present study differs from previous ones on hemineglect in two points: on the one hand, we used everyday photographs in order to represent more real life requirements. Previous studies used random arrays of letters (Husain et al., 2001; Karnath \& Niemeier, 2002; Karnath et al., 1998) or geometrical shapes (Sprenger et al., 2002). On the other hand, we presented a large number of images (i.e., 32 images). Some previous results (Husain et al., 2001; Karnath \& Niemeier, 2002; Karnath et al., 1998) were based on less than ten images. Increasing the number of images is likely to produce results that depend less on image content and may reflect systematic exploratory strategies more accurately.

\section{Subjects and methods}

Two groups of subjects were tested. A patient group of 15 inpatients from the neuropsychological rehabilitation center (11 males, mean age: 55.5 years; SD 9 years; range $43-$ 73 years) were examined, and an age-matched control group of 8 subjects (6 males, mean age: 48.5 years; SD 9.5 years). All patients had right hemisphere damage and showed clinical signs of acute left-sided visual hemineglect (e.g., bumping into objects in the left space). The subjects were examined within the first 2 months after brain lesion (ischemic or hemorrhagic stroke). All subjects gave written informed consent, and the study was approved by the ethical committee of the State of Bern and consistent with the latest Declaration of Helsinki.

Patients and control subjects were tested in a free visual exploration task. Thirty-two naturalistic color photographs of everyday scenes (a representative example is shown in Fig. 1) were displayed in a dark room on a cathode ray tube computer display (Samsung SyncMaster 959NF), with a

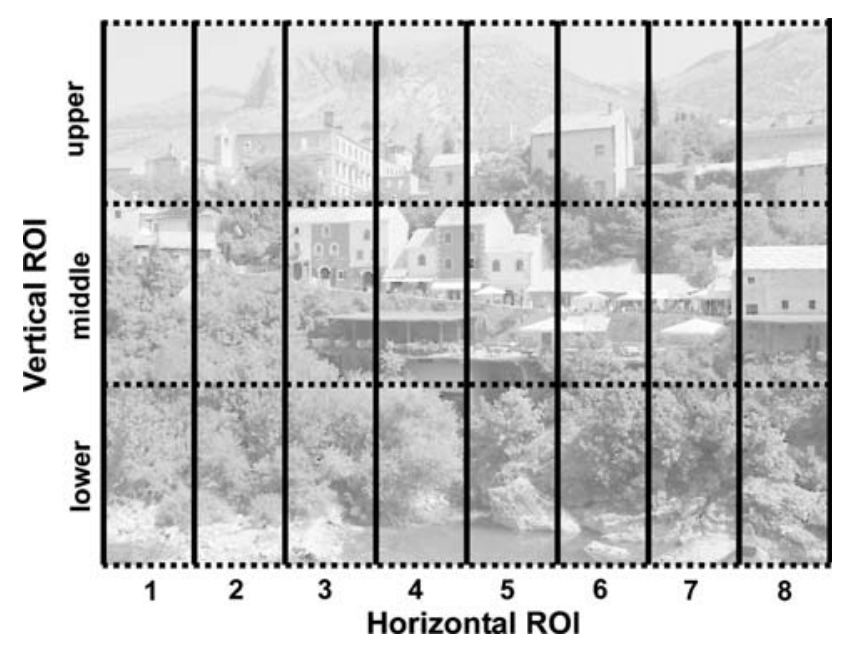

Fig. 1 Topographic analysis of the distribution of the cumulative fixation duration with definition of horizontal and vertical ROIs 
size of $36 \times 27 \mathrm{~cm}$ and a resolution of $800 \times 600$ pixels ( 24 bit color depth with a refresh rate of $85 \mathrm{~Hz}$ ). Each photograph was presented for $7 \mathrm{~s}$. A central fixation point was presented for $1 \mathrm{~s}$ before every image presentation. Subjects were instructed to fixate the central fixation point between image presentations.

Eye position was recorded with an infrared video-based tracking system (EyeLink $^{\mathrm{TM}}$, Sensomotoric Instruments $\mathrm{GmbH}$, Teltow, Germany). The system has a temporal resolution of $250 \mathrm{~Hz}$, a spatial resolution of $0.01^{\circ}$, and a gaze position accuracy of $0.5^{\circ}-1.0^{\circ}$, depending on participant's fixation accuracy during calibration. Minimal fixation duration was set at $100 \mathrm{~ms}$. As the system is equipped with a head movement compensation mechanism, no head fixation was necessary, and a chin rest was sufficient to secure a constant viewing distance of $70 \mathrm{~cm}$, resulting in a viewing angle of $29^{\circ} \times 22^{\circ}$. The system was calibrated twice before each block of images by means of two $3 \times 3$ point grid calibration sequences.

\section{Data analysis}

The eye movements were parsed into fixations and saccades in real time by the EyeLink system. Further data analysis was performed off-line. The following parameters were analyzed: (1) Cumulative fixation duration in each subject for a given condition [screen-half and regions of interest (ROI); see below]. (2) The direction of the first saccade, calculating for each subject the percentage of first leftward saccades. (3) Saccades were classified into left- or rightward saccades according to their horizontal left- or rightward component, and mean number and amplitude was calculated for each subject. (4) To evaluate visual fixation distribution, the screen was divided into eight identical, vertically oriented ROIs, each 100 pixels wide and in additional vertical ROIs each of 200 pixels height (i.e., upper, middle, and lower vertical ROI, see Fig. 1).

For statistical analysis of the distribution of the cumulative fixation duration, number and amplitude of saccades, a repeated-measures ANOVA was performed with the between-subject factor "neglect" (i.e., patient vs. control group) and within-subject factors "side" (left screen-half vs. right screen-half) or "ROI" and "saccade direction" (leftward and rightward saccades).

\section{Results}

Figure 2 shows the cumulative fixation duration for the left and right screen-half. Repeated-measures ANOVA yielded a significant main effect for "side" $[F(1,21)=9.42$, $P<0.01]$ and a significant interaction "side $\times$ neglect" with $[F(1,21)=13.34, P<0.01]$.

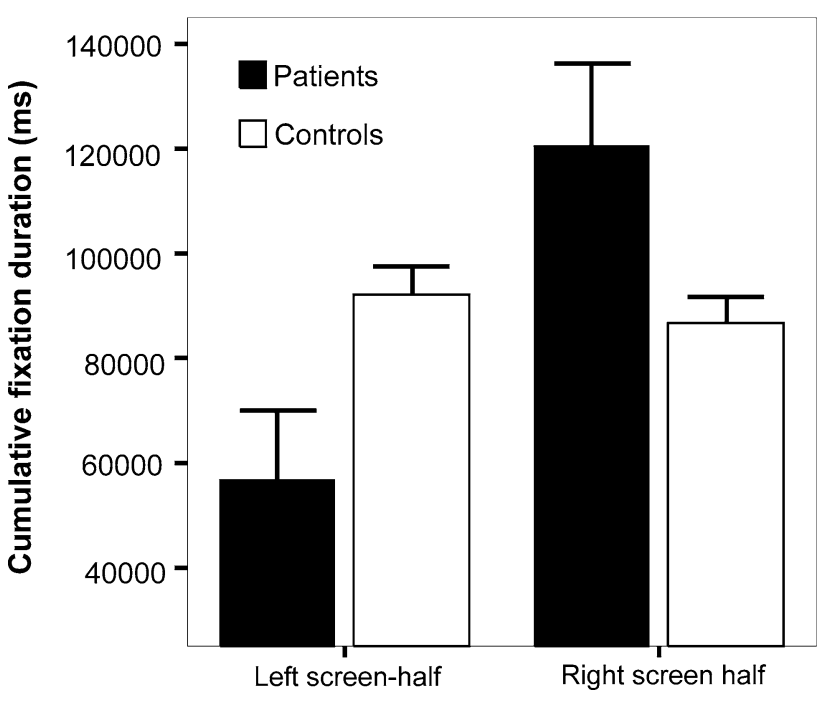

Fig. 2 Cumulative fixation duration in patients (black bars) with leftsided hemineglect and in control subjects. Errors bars: SEM

Post hoc testing showed significant difference between patients and controls for the left screen half (patients: mean 56,314 ms, controls: $92,001 \mathrm{~ms} ; P<0.001$ ), and the rightscreen half (patients: mean 120,209 ms, controls: $86,457 \mathrm{~ms} ; P<0.01)$.

The median percentage of first leftward saccade for patients and controls is shown in Fig. 3.

In the patient group, there was a significant reduction of the median percentage of first leftward saccade was $22 \%$ [interquartile range (IQR): 16\%] compared to the control

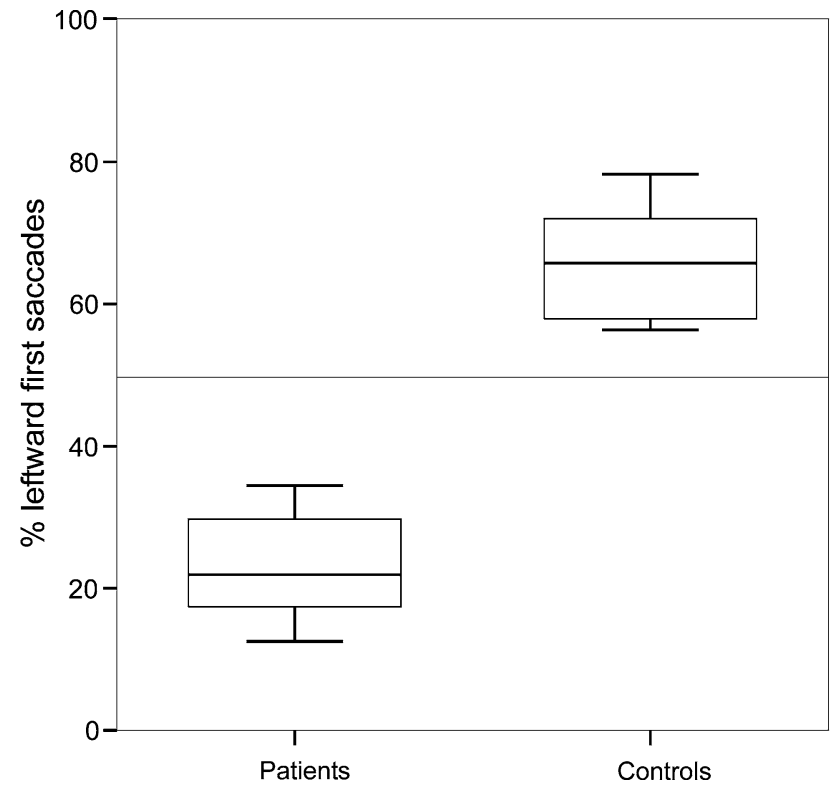

Fig. 3 Percentage of first saccades to the left side. There is a significant reduction of the percentage of leftward saccades in patients compared the controls $(P<0.01)$ 
Fig. 4 Mean saccade amplitude dependent from screen half and saccade direction. Patients (black bars) showed for both leftward and rightward saccades significantly reduced amplitudes compared the controls (open bars). Left- and rightward arrows indicate saccade direction. Error bars SEM
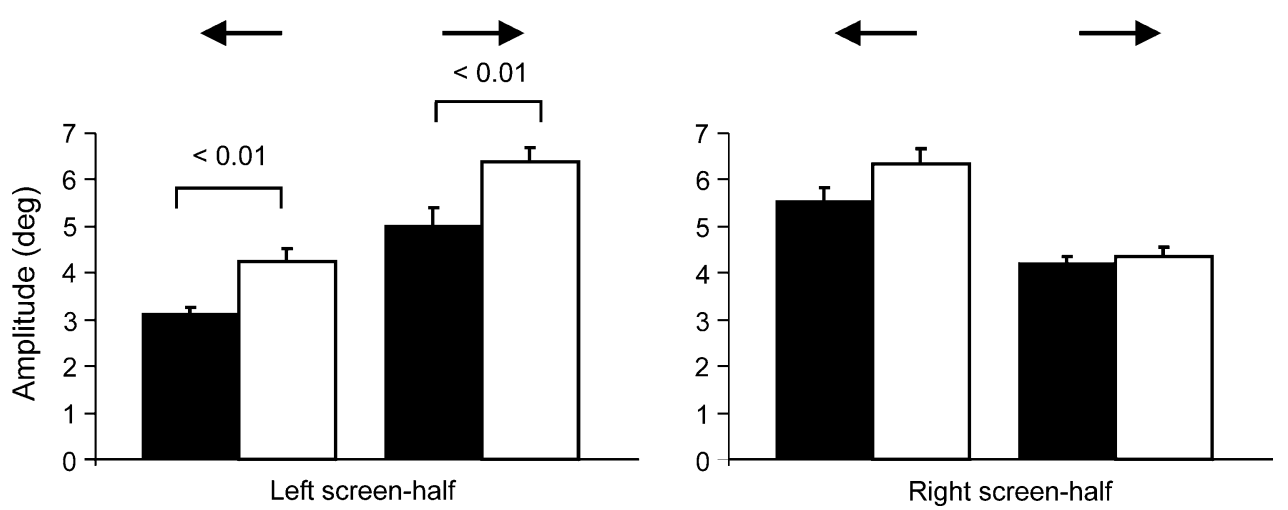

group: median 66\% (IQR: 16\%; Mann-Whitney $U$ test $P<0.01)$.

Concerning saccade amplitude, repeated-measures ANOVA yielded significant main effect for "side" $[F(1,21)=11.49, P<0.01]$. Significant interactions were found for "side $\times$ neglect" with $[F(1,21)=11.59, P<0.01]$ and for "side $\times$ saccade direction" with $[F(1,21)=174.80$, $P<0.001]$. There was no significant main effect for "saccade direction". The results are shown in Fig. 4. Post hoc testing showed significantly shorter saccade amplitudes in patients than in controls for the left screen half: in patients, mean amplitude was $3.1^{\circ}$ (SEM: $0.1^{\circ}$ ) for leftward saccades. In controls, mean leftward amplitude was $4.3^{\circ}$ $\left(0.3^{\circ}\right)$. For rightward saccades, patient's mean amplitude was $4.9^{\circ}\left(0.2^{\circ}\right)$, in controls mean rightward amplitude was $6.4^{\circ}(0.3)$.

Repeated-measures ANOVA for the number of saccades yielded significant main effect for "saccade direction" $[F(1,21)=11.62, P<0.01]$. The main factor "side" was not significant. Significant interactions were found for "saccade direction $\times$ neglect" with $[F(1,21)=14.43, P<0.001]$ and for "side $\times$ saccade direction" with $[F(1,21)=72.51$, $P<0.001]$.

The results are shown in Fig. 5. Post hoc testing between patients and controls were significant for all conditions: in the left screen-half, patients made on average 60 leftward saccades (SEM: 9 saccades) and controls on average139 (8). Patients made on average 212 (12) rightward saccades and controls 175 (8). In the right screen-half, mean number of leftward saccades in patients was 107 (12), in controls 167 (8). Mean number of rightward saccades in patients was 163 (16), in controls 120 (8).

The distribution of the cumulative fixation duration over horizontal ROI's is presented in Fig. 6. The repeated measures ANOVA including horizontal and vertical ROI's showed significant main effects for "horizontal ROI" $[F(7,147)=39.94, \quad P<0.001]$ and "vertical ROI" $[F(2,42)=348.36, \quad P<0.001]$. The interactions "neglect $\times$ horizontal ROI" $[F(7,147)=9.86, P<0.001]$ and "neglect $\times$ vertical ROI" $[F(2,42)=13.77, P<0.001]$ and the interaction "horizontal $\times$ vertical ROI" $[F(14,294)=28.40, P<0.001]$ were significant. Finally, the three times interaction "neglect $\times$ horizontal ROI $\times$ vertical ROI" $[F(14,294)=7.44, P<0.001]$ was also significant. Significant post hoc testing $(P<0.05)$ for the 3 times interaction is presented in Fig. 7.

Patients showed the typical bias towards the right side of the screen-half, with a reduction of cumulative fixation duration in the left screen-half and an increase in the right screen-half. When the cumulative fixation duration of patients was expressed as the percentage of the control group (i.e., $100 \%$ corresponds to the cumulative fixation duration of the control group), the right-sided bias was more evident (Fig. 6, open triangles, right $y$-axis). The bias was not linear shift but corresponded to a more complex curve with a maximum near the center of the right screen-half
Fig. 5 Mean number of saccades. Patients (black bars) were for all conditions significantly different compared to the controls (open bars). Left- and rightward arrows indicate saccade direction. Error bars SEM
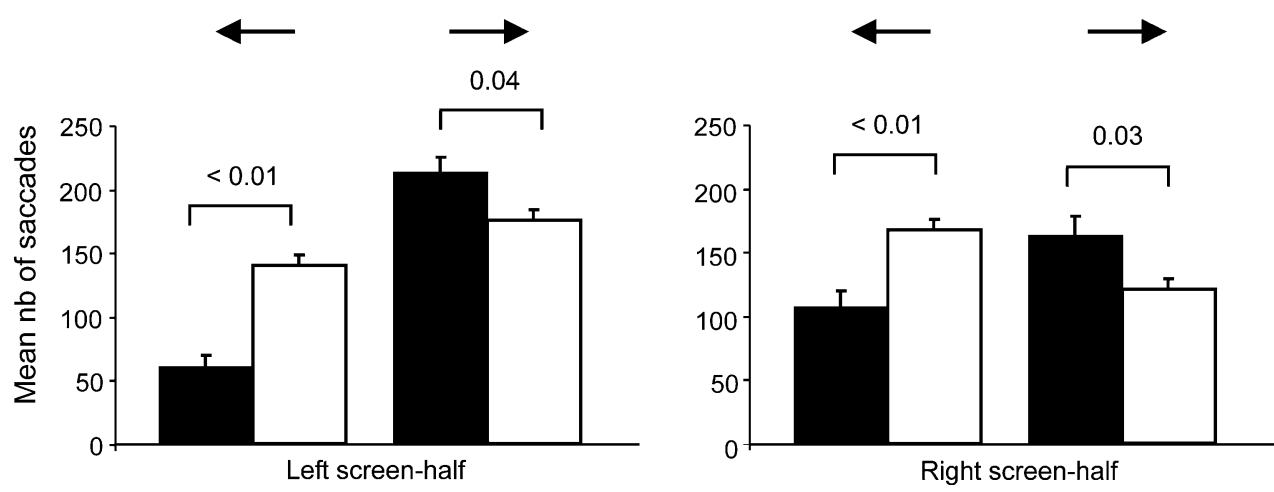


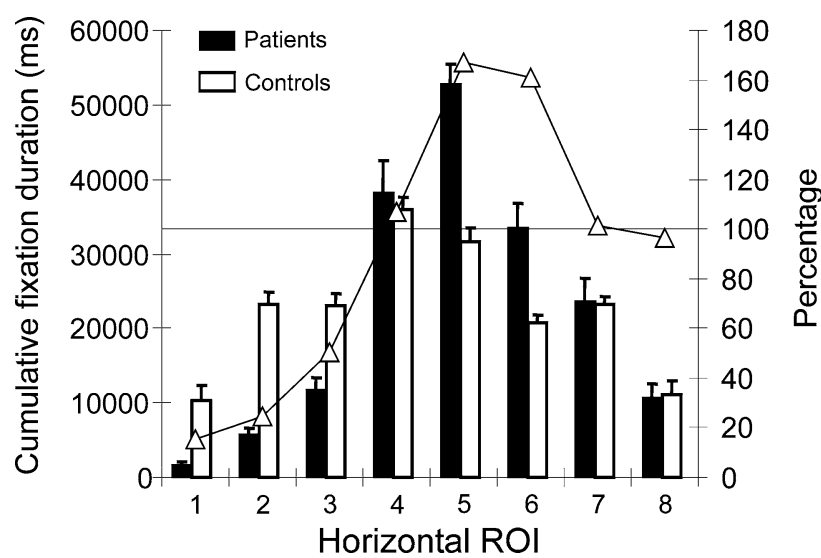

Fig. 6 Distribution of the cumulative fixation duration dependent of the horizontal ROI. Cumulative fixation duration in $\mathrm{ms}$ is presented in bars (black bars for patients, open bars for controls. Open triangles of the line plot present the percentage of cumulative fixation duration in the patient group $(100 \%$ corresponds to the cumulative fixation duration of the control group for a given ROI)

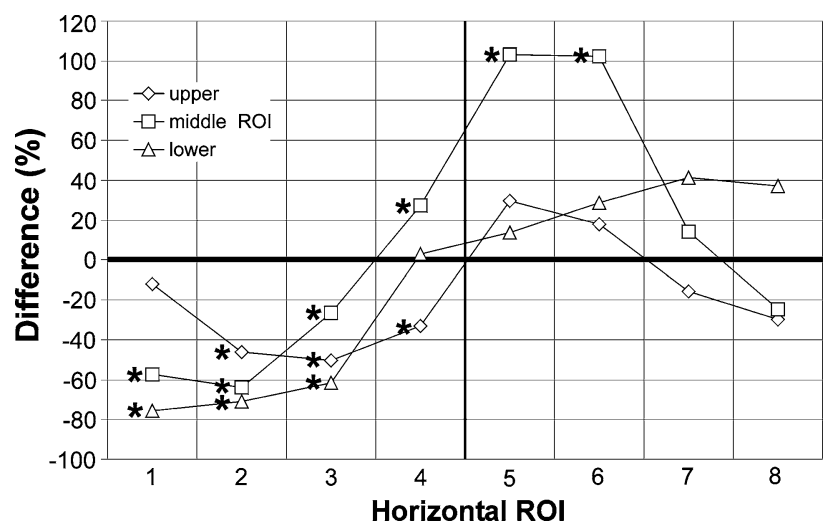

Fig. 7 Mean differences of the cumulative fixation duration in neglect patients (in percent of the control group), depending of horizontal and vertical ROI. The most important reduction of cumulative fixation duration was found for the lower ROI on the left side. Significant post hoc tests of the interaction "neglect $\times$ horizontal ROI $\times$ vertical ROI" are marked by asterisk

(ROI 5 and 6). Interestingly, the percentage of cumulative fixation duration in patients for more eccentric ROI's on the right screen-half was close to $100 \%$, i.e., similar to the control group. To present the results of the complex interaction of horizontal and vertical ROI on cumulative fixation duration, the difference between patients and controls was calculated for each ROI and expressed as percentage. Thus, a difference of $0 \%$ means the same cumulative fixation duration in patients as in controls. These results are shown in Fig. 7. In the left screen-half, the most important difference was found for the lower vertical ROI with significant differences to the controls for horizontal ROI 1 to 3 (the most eccentric ROI of the screen). For the middle vertical ROI, a similar reduction was found, however, less pronounced.
The overall rightward bias seems mainly to be caused by the cumulative fixation duration within the middle vertical ROI, since in the right screen-half, cumulative fixation duration in patients compared to controls was only significantly increased in the middle vertical ROI. Smallest differences were found in the upper vertical ROI.

\section{Discussion}

The aim of the current experimental study was to analyze eye movement behavior in patients with acute left-sided neglect during a free exploration task. The analysis of eye movement parameters such as cumulative fixation duration, saccade amplitude, or the numbers of saccades has been used in several studies to probe attention deficits in neglect patients (e.g., Ro, Rorden, Driver, \& Rafal, 2001; Olk, Harvey, \& Gilchrist, 2002; Pflugshaupt et al., 2004), since the pattern of exploratory eye movements has been taken as a strong index of attention distribution (Kowler, Anderson, Dosher, \& Blaser., 1995; Kustov \& Robinson, 1996).

The typical left-right bias in our neglect patients was demonstrated by several oculomotor parameters: cumulative fixation duration, direction of the first saccade, the number, direction, and amplitude of saccades. Furthermore, spatial distribution of the cumulative fixation duration demonstrated also an exploration bias in the vertical direction, compared to healthy controls.

Cumulative fixation duration describes the overall relevance of exploration behavior (e.g., Behrmann et al., 1997). Our patients explored the left screen-half approximately half time compared to the right screen-half, a finding, which was also observed by, e.g., Behrmann et al. The spatial distribution of cumulative fixation duration showed a left-right horizontal gradient, which was not linear. It has been proposed that the distribution of visual fixations may critically depend on the size of the visual array (Karnath \& Niemeier, 2002). When using smaller exploration fields (e.g., a visual angle of $45^{\circ}$ as in Behrmann et al., 1997), a more gradient-shaped distribution is found. A rotation of the distribution, however, is found when large exploration fields are used (e.g., a visual angle of $280^{\circ}$ as in Karnath et al., 1998).

Neglect patients made more first saccades towards the ipsilesional as opposed to the contralesional screen-half. Biases in early orienting have been reported in many studies with acute (e.g., Azouvi et al., 2002; Gainotti, D'Erme \& Bartolomeo, 1991; Mattingley, Bradshaw, Bradshaw, \& Nettleton, 1994; Olk et al., 2002) and residual neglect patients (Pflugshaupt et al., 2004; Mattingley et al., 1994; Olk et al., 2002).

The saccade amplitude during free exploration was also affected depending of the screen-half. In the left screenhalf, patients produced independently of saccade direction 
significantly smaller saccade amplitudes compared to the control group. Interestingly, in the right screen-half saccade amplitudes of neglect patients were not reduced, irrespective of saccade direction. For the number of saccades made during free exploration, we found direction-specific effects: the number of saccades was significantly reduced for leftward saccades and significantly increased for rightward saccades. Studies in neglect patients examining saccade planning towards unilaterally presented visual targets reported significantly decreased amplitude of contralesional saccades, but unaffected ipsilesional saccades (Heide \& Kömpf, 1998; Ptak, Schnider, Golay, \& Müri, 2007; Walker \& Findlay, 1996). Furthermore, neglect patients are often slower to initiate contralesional saccades or making small, multistep saccades (Walker \& Findlay, 1996; Behrmann, Ghiselli-Crippa \& Dimatteo, 2001). Amplitudes of left and right saccades are decreased when neglect patients made exploratory eye movements in the dark (Karnath \& Niemeier, 2002; Karnath et al., 1998). In visual search tasks (Niemeier \& Karnath, 2000), patients with neglect do not necessarily make fewer saccades in the contralesional direction, but the saccades are generally of small amplitude. Finally, saccade amplitude deficit in neglect may be most prominent for stimulus-driven or reflexive saccades than for volitional saccades (Niemeier \& Karnath, 2003).

A further important point we addressed in our study was the influence of neglect on fixation distribution in the vertical direction. To the best of our knowledge, there is no study analyzing fixation distribution in the vertical plane during a free exploration task. Compared to the control group, neglect patients showed the most prominent reduction of cumulative fixation duration (on average 80\%) in the lower leftmost ROI. Furthermore, there was a gradient in reduction between upper, middle, and lower ROI in the left screen-half.

In the more central ROI's of the left screen-half, the cumulative fixation duration increased rapidly to control values for the middle vertical ROI. Cumulative fixation duration within the middle vertical ROI seems mainly responsible for the increased of cumulative fixation duration in the right screen-half. In other words, the left-right bias observed in visual exploration behavior of neglect patients seems to be due to the increase of fixations in the middle vertical ROI. On the one hand, in the left screenhalf, the reduction of fixation distribution was prominent in the most eccentric lower ROI, but also significant reductions of cumulative fixation duration were found for the middle and upper vertical ROI. On the other hand, in the right screen-half a significant increase of the cumulative fixation duration was only observed for the middle vertical ROI.

The reduction of cumulative fixation duration in the lower most eccentric is interesting in view of several stud- ies showing reduced performance in the vertical direction in neglect patients. Halligan and Marshall (1989) found in patients with unilateral neglect, that these patients made most errors in the lower quadrant in a line bisection task, contralateral to the lesion. Their results suggest that the vertical position of a stimulus has a significant influence of performance in neglect. Pitzalis, Spinelli, and Zoccolotti (1997) were able to reproduce these results in their neglect patients. Furthermore, latencies of visually evoked potentials in the lower left quadrant were longer than those in the other quadrants.

Exploration behavior in neglect is also dependent on the size and type of the visual array (Karnath \& Niemeier, 2002), and in our study we used a relatively small exploration field with an angle of $29^{\circ}$. One might also argue that natural scenes such as landscapes have a horizontal bias, and the most salient elements are localized near the vertical middle of the screen. However, the distribution of fixations in the vertical direction was averaged over 32 different images that were not only landscapes. More importantly, such a distribution in the vertical plane was not observed in healthy controls. In conclusion, the results of our study show that in neglect patients fixation distribution of free exploration of natural scenes is not only influenced by the left-right bias in the horizontal direction but also by the vertical direction.

Acknowledgments The study was supported by SNF Grants 3200B0-116074/1 and 320000-108146.

\section{References}

Adair, J. C., Williamson, D. J., Jacobs, D. H., \& Heilman, K. M. (1995). Neglect of radial and vertical space: Importance of the retinotopic reference frame. Journal of Neurology, Neurosurgery, and Psychiatry, 58, 724-728.

Azouvi, P., Samuel, C., Louis-Dreyfus, A., Bernati, T., Bartolomeo, P., Beis, J. M., et al. (2002). Sensitivity of clinical and behavioural tests of spatial neglect after right hemisphere stroke. Journal of Neurology, Neurosurgery, and Psychiatry, 73, 160-166.

Barrett, A. M., Buxbaum, L. J., Coslett, H. B., Edwards, E., Heilman, K. M., Hillis, A. E., et al. (2006). Cognitive rehabilitation interventions for neglect and related disorders: Moving from bench to bedside in stroke patients. Journal of Cognitive Neuroscience, 18, 1223-1236.

Bartolomeo, P., \& Chokron, S. (1999a). Left unilateral neglect or right hyperattention? Neurology, 53, 2023-2027.

Bartolomeo, P., \& Chokron, S. (1999b). Egocentric frame of reference: Its role in spatial bias after right hemisphere lesions. Neuropsychologia, 37, 881-894.

Behrmann, M., Ghiselli-Crippa, T., \& Dimatteo, I. (2001). Impaired initiation but not execution of contralesional saccades in hemispatial neglect. Behavioral Neurology, 13, 39-60.

Behrmann, M., Watt, S., Black, S. E., \& Barton, J. J. (1997). Impaired visual search in patients with unilateral neglect: An oculographic analysis. Neuropsychologia, 35, 1445-1458.

Bisiach, E., Pizzamiglio, L., Nico, D., \& Antonucci, G. (1996). Beyond unilateral neglect. Brain, 119, 851-857. 
Buxbaum, L. J., Ferraro, M. K., Veramonti, T., Farne, A., Whyte, J., Ladavas, E., et al. (2004). Hemispatial neglect: Subtypes, neuroanatomy and disability. Neurology, 62, 749-756.

Cappelletti, M., Freeman, E. D., \& Cipolotti, L. (2007). The middle house or the middle floor: Bisecting horizontal and vertical mental number lines in neglect. Neuropsychologia, 45, 2989-3000.

Chatterjee, A. (1998). Motor minds and mental models in neglect. Brain and Cognition, 37, 339-349.

Chokron, S., \& Bartolomeo, P. (1997). Patterns of dissociation between left hemineglect and deviation of the egocentric reference. Neuropsychologia, 35, 1503-1508.

Corbetta, M., Akbudak, E., Conturo, T. E., Snyder, A. Z., Ollinger, J. M., Drury, H. A., et al. (1998). A common network of functional areas for attention and eye movements. Neuron, 21, 761-773.

Danckert, J., \& Ferber, S. (2006). Revisiting unilateral neglect. Neuropsychologia, 44, 987-1006.

Gainotti, G., D'Erme, P., \& Bartolomeo, P. (1991). Early orientation of attention toward the half space ipsilateral to the lesion in patients with unilateral brain damage. Journal of Neurology, Neurosurgery, and Psychiatry, 54, 1082-1089.

Halligan, P. W., \& Marshall, J. C. (1989). Is neglect (only) lateral? A quadrant analysis of line cancellation. Journal of Clinical and Experimental Neuropsychology, 11, 793-798.

Halligan, P. W., \& Marshall, J. C. (1991). Spatial compression in visual neglect: A case study. Cortex, 27, 623-629.

Heide, W., \& Kömpf, D. (1998). Combined deficits of saccades and visuo-spatial orientation after cortical lesions. Experimental Brain Research, 23, 164-171.

Himmelbach, M., Erb, M., \& Karnath, H.-O. (2006). Exploring the visual world: The neural substrate of spatial orienting. Neuroimage, $32,1747-1759$.

Husain, M., \& Kennard, C. (1996). Visual neglect associated with frontal lobe infarction. Journal of Neurology, 243, 652-657.

Husain, M., \& Rorden, C. (2003). Non-spatially lateralized mechanisms in hemispatial neglect. Nature Reviews Neuroscience, 4, 26-36.

Husain, M., Mannan, S., Hodgson, T., Wojciulik, E., Driver, J., \& Kennard, C. (2001). Impaired spatial working memory across saccades contributes to abnormal search in parietal neglect. Brain, 124, 941-952.

Ignashchenkova, A., Dicke, P. W., Haarmeier, T., \& Thier, P. (2004). Neuronspecific contribution of the superior colliculus to overt and covert shifts of attention. Nature Neuroscience, 7, 56-64.

Karnath, H.-O. (1997). Spatial orientation and the representation of space with parietal lobe lesions. Philosophical Transaction of the Royal Society, London B, 352, 1411-1419.

Karnath, H.-O. (1998). Perception of horizontal distances in patients with spatial neglect. Experimental Brain Research, 23, 190-191.

Karnath, H.-O., Ferber, S., \& Himmelbach, M. (2001). Spatial awareness is a function of the temporal not the posterior parietal lobe. Nature, 411, 950-953.

Karnath, H.-O., \& Fetter, M. (1995). Ocular space exploration in the dark and its relation to subjective and objective body orientation in neglect patients with parietal lobe lesions. Neuropsychologia, 33, 371-377.

Karnath, H.-O., Fetter, M., \& Dichgans, J. (1996). Ocular exploration of space as a function of neck proprioceptive and vestibular input. Observations in normal subjects and patients with spatial neglect after parietal lesions. Experimental Brain Research, 109, 333-342.

Karnath, H.-O., Fruhmann-Berger, M., Küker, W., \& Rorden, C. (2004). The anatomy of spatial neglect based on voxelwise statistical analysis: A Study of 140 Patients. Cerebral Cortex, 14, $1162-1172$.

Karnath, H.-O., Himmelbach, M., \& Rorden, C. (2002). The subcortical anatomy of human spatial neglect: Putamen, caudate nucleus and pulvinar. Brain, 125, 350-360.
Karnath, H.-O., \& Niemeier, M. (2002). Task-dependent differences in the exploratory behavior of patients with spatial neglect. Neuropsychologia, 40, 1577-1585.

Karnath, H.-O., Niemeier, M., \& Dichgans, J. (1998). Space exploration in neglect. Brain, 121, 2357-2367.

Karussis, D., Leker, R. R., \& Abramsky, O. (2000). Cognitive dysfunction following thalamic stroke: A study of 16 cases and review of the literature. Journal of The Neurological Sciences, 172, 25-29.

Kinsbourne, M. (1993). Orientational bias model of unilateral neglect: Evidence from attentional gradients within hemispace. In I. H. Robertson \& J. C. Marshall (Eds.), Unilateral neglect: Clinical and experimental studies (pp. 63-86). Hove (UK): Lawrence Erlbaum Associates.

Kowler, E., Anderson, E., Dosher, B., \& Blaser, E. (1995). The role of attention in the programming of saccades. Vision Research, 35, 1897-1916.

Kustov, A. A., \& Robinson, D. L. (1996). Shared neural control of attention shifts and eye movements. Nature, 384, 74-77.

Losier, B. J. W., \& Klein, R. M. (2001). A review of the evidence for a disengage deficit following parietal lobe damage. Neuroscience and Behavioral Reviews, 25, 1-13.

Malhotra, P., Coulthard, E., \& Husain, M. (2006). Hemispatial neglect, balance and eye-movement control. Current Opinion in Neurology, 19, 14-20.

Mark, V. W., \& Heilman, K. M. (1997). Diagonal neglect on cancellation. Neuropsychologia, 35, 1425-1436.

Marshall, J. C., Halligan, P. W., \& Robertson, I. H. (1993). Contemporary theories of unilateral neglect: A critical review. In I. H. Robertson \& J. C. Marshall (Eds.), Unilateral neglect: Clinical and experimental studies (pp. 311-329). Hove: Lawrence Erlbaum Associates.

Mattingley, J. B., Bradshaw, J. L., Bradshaw, J. A., \& Nettleton, N. C. (1994). Residual rightward attentional bias after apparent recovery from right hemisphere damage: Implications for a multicomponent model of neglect. Journal of Neurology, Neurosurgery, and Psychiatry, 57, 597-604.

Mennemeier, M., Wertman, E., \& Heilman, K. M. (1992). Neglect of near peripersonal space. Brain, 115, 37-50.

Mort, D. J., \& Kennard, C. (2003). Visual search and its disorders. Current Opinion in Neurology, 16(1), 51-57.

Mort, D. J., Malhotra, P., Mannan, S. K., Rorden, C., Pambakian, A., Kennard, C., et al. (2003). The anatomy of visual neglect. Brain, 126, 1986-1997.

Niemeier, M., \& Karnath, H.-O. (2000). Exploratory saccades show no directionspecific deficit in neglect. Neurology, 54, 515-518.

Niemeier, M., \& Karnath, H.-O. (2003). Stimulus-driven and voluntary saccades are coded in different coordinate systems. Current Biology, 13, 585-589.

Nobre, A. C., Gitelman, D. R., Dias, E. C., \& Mesulam, M. M. (2000). Covert visual spatial orienting and saccades: Overlapping neural systems. NeuroImage, 11, 210-216.

Olk, B., Harvey, M., \& Gilchrist, I. D. (2002). First saccades reveal biases in recovered neglect. Neurocase, 8, 306-313.

Parton, A., Malhotra, P., \& Husain, M. (2004). Hemispatial neglect. Journal of Neurolgy, Neurosurgery, and Psychiatry, 75, 13-21.

Pflugshaupt, T., Bopp-Almoslochner, S., Heinemann, D., Mosimann, U. P., von Wartburg, R., Nyffeler, T., et al. (2004). Residual oculomotor and exploratory deficits in patients with recovered hemineglect. Neuropsychologia, 42, 1203-1211.

Pitzalis, S., Spinelli, D., \& Zoccolotti, P. (1997). Vertical neglect: Behavioral and electrophysiological data. Cortex, 33, 679-688.

Posner, M. I., Walker, J. A., Friedrich, F. J., \& Rafal, R. D. (1984). Effects of parietal injury on covert orienting of attention. The Journal of Neuroscience, 4, 1863-1874.

Ptak, R., Schnider, A., Golay, L., \& Müri, R. M. (2007). A non-spatial bias favouring fixated stimuli revealed in patients with spatial neglect. Brain, 130, 3211-3222. 
Rapcsak, S. Z., Cimino, C. R., \& Heilman, K. M. (1988). Altitudinal neglect. Neurology, 38, 277-281.

Richard, C., Rousseaux, M., \& Honoré, J. (2005). The egocentric reference deviation of neglect patients is influenced by visuospatial attention. Neuropsychologia, 43, 1784-1791.

Ro, T., Rorden, C., Driver, J., \& Rafal, R. (2001). Ipsilesional biases in saccades but not perception after lesions of the human inferior parietal lobule. Journal of Cognitive Neuroscience, 13, 920-929.

Shelton, P. A., Bowers, D., \& Heilman, K. M. (1990). Peripersonal and vertical neglect. Brain, 113, 191-205.

Smania, N., Martini, M. C., Gambina, G., Tomelleri, G., Palamara, A., Natale, E., et al. (1998). The spatial distribution of visual attention in hemineglect and extinction patients. Brain, 121, $1759-1770$.

Sprenger, A., Kömpf, D., \& Heide, W. (2002). Visual search in patients with left visual hemineglect. Progress in Brain Research, 140, 395-416.

Treisman, A. M., \& Gelade, G. (1980). A feature-integration theory of attention. Cognitive Psychology, 12, 97-136.

Vallar, G. (2001). Extrapersonal visual unilateral spatial neglect and its neuroanatomy. NeuroImage, 14, 52-58.

Walker, R., \& Findlay, J. M. (1996). Saccadic eye movement programming in unilateral neglect. Neuropsychologia, 34, 493-508. 